Mathematics Division, National Center for Theoretical Sciences at Taipei NCTS/TPE-Math Technical Report 2007-004

\title{
Half-Skyrmions and Spike-Vortex Solutions of Two-Component Nonlinear Schrödinger Systems
}

\author{
Tai-Chia Lin ${ }^{* \dagger}$ and Juncheng Wei ${ }^{\ddagger}$
}

\begin{abstract}
Recently, skyrmions with integer topological charges have been observed numerically but have not yet been shown rigorously on two-component systems of nonlinear Schrödinger equations (NLSE) describing a binary mixture of Bose-Einstein condensates (cf. [2] and 25]). Besides, half-skyrmions characterized by half-integer topological charges can also be found in the nonlinear $\sigma$ model which is a model of the Bose-Einstein condensate of the Schwinger bosons (cf. [18]). Here we prove rigorously the existence of half-skyrmions which may come from a new type of soliton solutions called spike-vortex solutions of two-component systems of NLSE on the entire plane $\mathbb{R}^{2}$. These spike-vortex solutions having spikes in one component and a vortex in the other component may form half-skyrmions. By Liapunov-Schmidt reduction process, we may find spike-vortex solutions of two-component systems of NLSE.
\end{abstract}

\section{Introduction}

Spikes and vortices are important phenomena in one-component nonlinear Schrödinger equations (NLSE) having applications in many physical problems, especially in Bose-Einstein condensation. In the last two decades, there have been many analytical works on both spikes and vortices, respectively. One may refer to [19] for a good survey on spikes, and [1], [11] and 21] for survey on vortices. Recently, a double condensate i.e. a binary mixture of Bose-Einstein condensates in two different hyperfine states has been observed and described by two-component

\footnotetext{
*Department of Mathematics, National Taiwan University, Taipei, Taiwan $106 . \quad$ email : tclin@math.ntu.edu.tw

${ }^{\dagger}$ National Center for Theoretical Sciences,Taipei Office

${ }_{\ddagger}^{\ddagger}$ Department of Mathematics, The Chinese University of Hong Kong, Shatin, Hong Kong. email: wei@math.cuhk.edu.hk
} 
systems of NLSE (cf. [22]). It would be possible to find spike and vortex solutions from twocomponent systems of NLSE. However, until now, there is no result to deal with spike-vortex solutions having spikes in one component and a vortex in the other component. In this paper, we want to find such solutions and investigate the interaction of spikes and vortices.

Ordinary skyrmions being of topological solitons resemble polyhedral shells which look like closed loops, possibly linked or knotted. Besides their intrinsic fundamental interest, skyrmions have important applications in nuclear physics (cf. [17]), and analogous structures are postulated for early universe cosmology (cf. [4]). To get a skyrmion, one may consider the multicomponent wave function which may introduce the extra internal degrees of freedom and result in a nontrivial structure characterized by topological charges. For two-dimensional Skyrme model, skyrmions have been investigated by the method of concentration-compactness (cf. [13]). In a double condensate, skyrmions with integer topological charges have been observed by numerical simulations on two-component systems of NLSE (cf. [2] and [25]). Besides, halfskyrmions characterized by half-integer topological charges can also be found in the nonlinear $\sigma$ model which may describe the Bose-Einstein condensate of the Schwinger bosons (cf. [18]). Here we prove rigorously the existence of half-skyrmions in a double condensate using spikevortex solutions of two-component systems of NLSE.

To get spike-vortex solutions, we study soliton solutions of time-dependent two-component systems of NLSE as follows:

$$
-\sqrt{-1} \frac{\partial \psi_{j}}{\partial t}=\triangle \psi_{j}+\sum_{i=1}^{2} \beta_{i j}\left|\psi_{i}\right|^{2} \psi_{j}, \quad \text { for } x \in \mathbb{R}^{n}, t>0, j=1,2,
$$

where the spatial dimension $n=2$ and $\psi_{j}=\psi_{j}(x, t) \in \mathbb{C}$ for $j=1,2$. The system (1.1) is a standard model to describe a double condensate. Physically, $\psi_{j}$ 's are the corresponding complex-valued wave functions, and the coefficients $\beta_{i j} \sim-a_{i j}$ for $i, j=1,2$, where $a_{j j}$ 's and $a_{12}=a_{21}$ are the intraspecies and interspecies scattering lengths, respectively. When the spatial dimension is one, i.e. $n=1$, it is well-known that the system (1.1) is integrable, and there are many analytical and numerical results on soliton solutions of coupled nonlinear Schrödinger equations (e.g. [7], 8], 9]). Recently, from physical experiments (cf. [3]), even three-dimensional solitons have been observed in Bose-Einstein condensates. It is natural to believe that there are two-dimensional (i.e. $n=2$ ) solitons in double condensates. However, when the spatial dimension is two, the system (1.1) becomes non-integrable and has only few results on twodimensional solitons. This may lead us to study two-dimensional soliton solutions of the system (1.1) and find different types of solitons.

In the vicinity of a Feshbach resonance, scattering lengths $a_{i j}$ 's depend sensitively on the magnitude of an externally applied magnetic field (cf. 23] and [24]), allowing the magnitude and sign of $\beta_{i j}$ 's to be tuned to any value. Generically, when both $\beta_{j j}$ 's are positive, the system (1.1) is of self-focusing and has bright soliton solutions on the associated two components. On the other hand, when both $\beta_{j j}$ 's are negative, the system (1.1) is of self-defocusing and has dark soliton solutions on the associated two components. Here we have interest on the case that $\beta_{11}$ and $\beta_{22}$ have opposite signs which may result in a new type of soliton solutions called spikevortex solutions of the system (1.1) i.e. one component has spikes and the other component has a vortex. Furthermore, we may obtain half-skyrmions by these spike-vortex solutions. 
To obtain soliton solutions of the system (1.1), we set

$$
\psi_{j}(x, t)=e^{\sqrt{-1} \lambda_{j} t} \cdot u_{j}(x), \quad u_{j} \in \mathbb{C}, j=1,2 .
$$

Substituting (1.2) into (1.1), we may obtain a two-component system of semilinear elliptic partial differential equations given by

$$
\left\{\begin{array}{l}
\triangle u_{1}-\lambda_{1} u_{1}+\beta_{11}\left|u_{1}\right|^{2} u_{1}+\beta_{12} u_{1}\left|u_{2}\right|^{2}=0, \\
\triangle u_{2}-\lambda_{2} u_{2}+\beta_{22}\left|u_{2}\right|^{2} u_{2}+\beta_{12}\left|u_{1}\right|^{2} u_{2}=0,
\end{array}\right.
$$

where $\beta_{12}$ is the coupling constant. In [15]-[16], we studied the ground state solutions of (1.3) for the case that $\beta_{11}>0, \beta_{22}>0$ and $u_{j}$ 's are positive functions. Namely, we investigated the following problem:

$$
\left\{\begin{array}{l}
\triangle u-\lambda_{1} u+\beta_{11} u^{3}+\beta_{12} u v^{2}=0 \\
\triangle v-\lambda_{2} v+\beta_{22} v^{3}+\beta_{12} u^{2} v=0 \\
u, v>0, u, v \in H^{1}\left(\mathbb{R}^{n}\right) .
\end{array}\right.
$$

Due to each $\lambda_{j}>0$ and $\beta_{j j}>0$, both $u$ and $v$ components have attractive self-interaction which may let spikes occur in these two components. One the other hand, when $n=2, \lambda_{j}<0$ and $\beta_{j j}<0, j=1,2$, and $u_{j}$ 's are complex-valued solutions of (1.3), vortices may exist in both $u_{1}$ and $u_{2}$ components (cf. [12]).

In this paper, we study the case that $n=2, \lambda_{1}, \beta_{11}>0, \lambda_{2}, \beta_{22}<0$, and $u_{1}$ is positive but $u_{2}$ is complex-valued function. Without loss of generality, we may assume that

$$
\lambda_{1}=-\lambda_{2}=\beta_{11}=-\beta_{22}=1 \text {. }
$$

Namely, we study the following system:

$$
\begin{cases}\triangle u-u+u^{3}+\beta u|v|^{2}=0 & \text { in } \mathbb{R}^{2}, \\ \triangle v+v-|v|^{2} v+\beta u^{2} v=0 & \text { in } \mathbb{R}^{2},\end{cases}
$$

where $u>0$ and $v \in \mathbb{C}$. To get skyrmions, a defining property of the skyrmion is that the atomic field approaches a constant value at spatial infinity (cf. [26]). Hence we may set boundary conditions of the system (1.5) as follows: $u(x) \rightarrow 0$ and $|v(x)| \rightarrow 1$ as $|x| \rightarrow \infty$.

As $\beta=0$, the first equation of the system (1.5) becomes a standard nonlinear Schrödinger equation given by

$$
\triangle u-u+u^{3}=0, \quad u \in H^{1}\left(\mathbb{R}^{2}\right)
$$

which has a unique least-energy spike solution $w=w(r), r=|x|$ satisfying $w^{\prime}(r)<0$ for $r>0$ and

$$
w(r)=A_{0} r^{-\frac{1}{2}} e^{-r}\left[1+O\left(\frac{1}{r}\right)\right], \quad w^{\prime}(r)=-A_{0} r^{-\frac{1}{2}} e^{-r}\left[1+O\left(\frac{1}{r}\right)\right] .
$$

Actually, this is also a typical spike solution of nonlinear Schrödinger equations. On the other hand, as $\beta=0$, the second equation of the system (1.5) can be written as

$$
\triangle v+v-|v|^{2} v=0, \quad v=v(z) \in \mathbb{C} \quad \text { for } z \in \mathbb{C} \cong \mathbb{R}^{2},
$$


which is of conventional Ginzburg-Landau equations (cf. [1]) having a symmetric vortex solution of degree $d \in \mathbb{Z} \backslash\{0\}$ with the following form

$$
v_{d}(z)=S_{d}(r) e^{\sqrt{-1} d \theta}
$$

where $S_{d}(r)$ satisfies

$$
\left\{\begin{array}{l}
S_{d}^{\prime \prime}+\frac{1}{r} S_{d}^{\prime}-\frac{d^{2}}{r^{2}} S_{d}+S_{d}-S_{d}^{3}=0 \\
S_{d}(0)=0, S_{d}(+\infty)=1,
\end{array}\right.
$$

and

$$
S_{d}^{\prime}(r)>0, \quad S_{d}(r)=1-\frac{d^{2}}{2 r^{2}}+O\left(\frac{1}{r^{4}}\right) \quad \text { as } r \rightarrow+\infty .
$$

Here we want to prove that when $\beta$ increases or decreases, there exist spike-vortex solutions of the system (1.5). This may become the first paper to illustrate such solutions of two-component nonlinear Schrödinger systems.

The main purpose of this paper is to construct a spike-vortex solution $(u, v)$ of the system (1.5) such that $u \sim w$ and $v \sim v_{d}$. Actually, the main difficulty of this paper is to study the interaction of typical spike and vortex solutions. Our first result shows that even a weak repulsive interaction $(\beta>0$ being small) can produce abundant bound states by solving the system (1.5). More precisely, we have

THEOREM 1.1. Let $n=2, d \in \mathbb{N}$ and $k \geq 2$ satisfy

(i) $k \geq 2$ is any positive integer if $d=1$,

(ii) $2(d-1) \not \equiv 0 \bmod k$ i.e. there does not exist any integer $\mu$ such that $2(d-1)=k \mu$ if $d \geq 2$.

Then for $\beta>0$ sufficiently small, the problem (1.5) has a solution $\left(u_{\beta}, v_{\beta}\right)$ satisfying $u_{\beta}(z)>0$ for $z \in \mathbb{C}, v_{\beta}(0)=0$ with degree $d$, and $u_{\beta}(z) \rightarrow 0,\left|v_{\beta}(z)\right| \rightarrow 1$ as $|z| \rightarrow \infty$. Moreover, as $\beta \rightarrow 0+,\left(u_{\beta}, v_{\beta}\right)$ has the following asymptotic form

$$
\left\{\begin{array}{l}
u_{\beta}(z)=\sum_{j=1}^{k} w\left(z-\xi_{j}^{\beta}\right)+O(|\beta|), \\
v_{\beta}(z)=S_{d}(r) e^{\sqrt{-1} d \theta} e^{\sqrt{-1} \psi_{\beta}(z)}, \psi_{\beta}(z)=O(|\beta|) \in \mathbb{C},
\end{array}\right.
$$

where $w$ is the unique radial solution of (1.6),$\left\langle\xi_{1}^{\beta}, \ldots, \xi_{k}^{\beta}\right\rangle$ forms a regular $k$-polygon with

$$
\xi_{j}^{\beta}=l_{\beta} e^{\sqrt{-1} \frac{2 \pi(j-1)}{k}}, j=1, \ldots, k,
$$

and $l_{\beta} \rightarrow+\infty$ as $\beta \rightarrow 0+$ satisfying $l_{\beta}=\hat{l}_{\beta}+O(1)$, where $\hat{l}_{\beta}$ satisfies

$$
\hat{l}_{\beta}^{\frac{5}{2}} e^{-2 \hat{l}_{\beta} \sin \frac{\pi}{k}}=\beta .
$$

A picture of $\left(u_{\beta}, v_{\beta}\right)$ is given by 


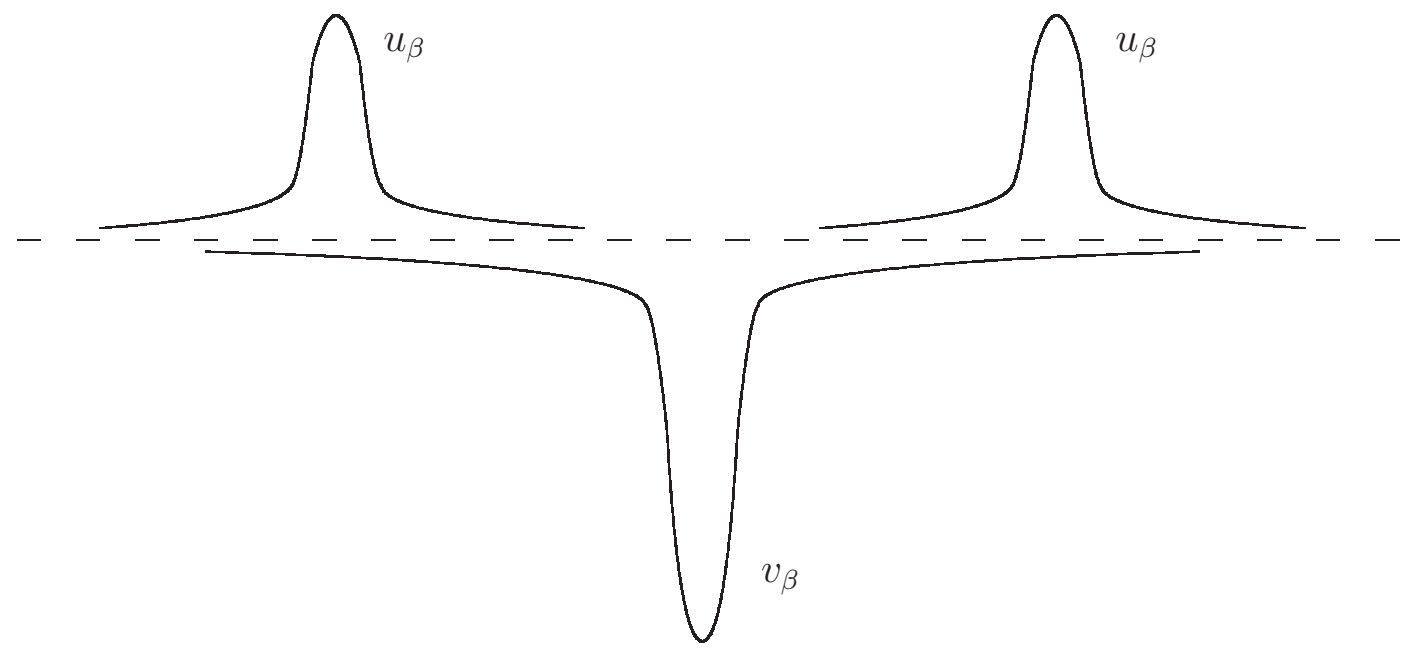

In [15]-[16], the positive sign of $\beta$ may give inter-component attraction when the intercomponent interaction is only for spikes. Conversely, the positive sign of $\beta$ may contribute inter-component repulsion when the inter-component interaction is for spikes and a vortex. The inter-component repulsion between $u$ and $v$ components may balance with self-attraction in $u$-component so a new kind of soliton solutions called spike-vortex solutions of two-component nonlinear Schrödinger systems can be obtained in Theorem 1.1.

For $\beta<0$, we may consider the radial solution of (1.5) given by

$$
u=u(r), \quad v=f(r) e^{\sqrt{-1} d \theta},
$$

satisfying

$$
\begin{cases}u^{\prime \prime}+\frac{1}{r} u^{\prime}-u+u^{3}+\beta f^{2} u & =0, \quad \forall r>0 \\ f^{\prime \prime}+\frac{1}{r} f^{\prime}-\frac{d^{2}}{r^{2}} f+f-f^{3}+\beta u^{2} f=0, & \forall r>0 \\ u^{\prime}(0)=0, u(+\infty)=0 & \\ f(0)=0, f(+\infty)=1, & \end{cases}
$$

where $d \in \mathbb{N}$ and $(r, \theta)$ is the polar coordinates in $\mathbb{R}^{2}$. Then we have the following existence theorem:

THEOREM 1.2. Assume that $n=2$ and $\beta<0$. Then the problem (1.5) has radially symmetric solutions of the following form:

$$
u=u(r), \quad v=f(r) e^{\sqrt{-1} d \theta}
$$

where $u(r)$ is strictly decreasing, $f(r)$ is strictly increasing and $(u, f)$ satisfies (1.16).

\section{Remarks:}

1. It is easy to see that the solution $(u(r), f(r))$ is unique for the system (1.16) if $\beta$ is small enough. It is an interesting question to study the uniqueness for general $\beta<0$.

2. Note that the solution $\left(u(r), f(r) e^{\sqrt{-1} d \theta}\right)$ is not a global minimizer for the corresponding energy functional of (1.5) since the equation of $u$ is superlinear. It is conjectured that there 
exists a least energy solution (see definitions in [16]) satisfying $E\left[u(r), f(r) e^{\sqrt{-1} d \theta]} \leq E[u, v]\right.$ for any solution $(u, v)$ of $(1.5)$ with $\operatorname{deg}(v)=d$. Here the corresponding energy functional is defined as

$$
E[u, v]=\frac{1}{2} \int_{\mathbb{R}^{2}}\left(|\nabla u|^{2}+u^{2}\right)-\frac{1}{4} \int_{\mathbb{R}^{2}} u^{4}+\frac{1}{2} \int_{\mathbb{R}^{2}}|\nabla v|^{2}+\frac{1}{4} \int_{\mathbb{R}^{2}}\left(1-|v|^{2}\right)^{2}-\frac{\beta}{2} \int_{\mathbb{R}^{2}} u^{2}|v|^{2} .
$$

To get half-skyrmions, we may define a $S^{2}$-valued map

$$
\vec{n} \equiv \frac{1}{\sqrt{u^{2}+v_{1}^{2}+v_{2}^{2}}}\left(\begin{array}{l}
v_{1} \\
v_{2} \\
u
\end{array}\right)
$$

where $\left(u, v_{1}+\sqrt{-1} v_{2}\right)$ is the spike-vortex solution obtained in Theorem 1.1 and 1.2. Generically, the topological charge of $S^{2}$-valued maps is defined by (cf. [6])

$$
Q=\frac{1}{4 \pi} \int_{\mathbb{R}^{2}} \vec{n} \cdot\left(\partial_{x} \vec{n} \wedge \partial_{y} \vec{n}\right) d x d y
$$

Then we have

THEOREM 1.3. The $S^{2}$-map defined by (1.19) is of half-skyrmions with topological charge $\frac{d}{2}$.

Throughout the rest of the paper, we assume that

$$
\hat{l}_{\beta}-\gamma<l<\hat{l}_{\beta}+\gamma
$$

for some suitable $\gamma$. Note that

$$
\hat{l}_{\beta}=\frac{1}{2 \sin \frac{\pi}{k}} \log \frac{1}{\beta}+c_{k} \log \log \frac{1}{\beta}+O(1)
$$

where $c_{k}$ is constant depending on $k$ only. Besides, unless otherwise stated, the letter $C$ will always denote various generic constants which are independent of $\beta$, especially for $\beta$ sufficiently small. The constant $\alpha \in\left(0, \frac{1}{2}\right)$ is a fixed small constant.

The rest of this paper is organized as follows: In Section 2, we introduce useful properties about the spike solution $w$ and the symmetric vortex solution $v_{d}$. In Section 3, we define the approximate solutions of spike-vortex solutions and derive the associated error estimates. In Section 4, we use Liapunov-Schmidt reduction process to find spike-vortex solutions. Then we may complete the proof of Theorem 1.1 and 1.2 in Section 5 and 6, respectively. Finally, we give the proof of Theorem 1.3 in Section 7 .

Acknowledgments: The research of the first author is partially supported by a research Grant from NSC of Taiwan. The research of the second author is partially supported by an Earmarked Grant from RGC of Hong Kong. 


\section{Properties of Spikes and Vortex}

We recall some properties of $w$ and $S_{d}(r) e^{\sqrt{-1} d \theta}$, where $|d|=1$ or $|d|>1$ and $2(d-1) \not \equiv 0$ $\bmod k$. Let

$$
\left\{\begin{array}{l}
L_{1}[\phi]=\Delta \phi-\phi+3 w^{2} \phi \\
L_{2}[\psi]=\Delta \psi+\psi-S_{d}^{2} \psi-2 \operatorname{Re}\left(S_{d}(r) e^{-\sqrt{-1} d \theta} \psi\right) S_{d}(r) e^{\sqrt{-1} d \theta}
\end{array}\right.
$$

for $\phi$ is a real-valued function and $\psi$ is a complex-valued function. For convenience, we may define the conjugate operator of $L_{2}$ by

$$
\hat{L}_{2}:=e^{-\sqrt{-1} d \theta} L_{2} e^{\sqrt{-1} d \theta}
$$

Then by simple computations, it is easy to check that

$$
\hat{L}_{2}\left[\psi_{1}+\sqrt{-1} \psi_{2}\right]=\hat{L}_{2,1}\left[\psi_{1}, \psi_{2}\right]+\sqrt{-1} \hat{L}_{2,2}\left[\psi_{1}, \psi_{2}\right],
$$

for $\psi_{1}$ and $\psi_{2}$ are real-valued functions, where

$$
\begin{gathered}
\hat{L}_{2,1}\left[\psi_{1}, \psi_{2}\right]=\Delta \psi_{1}+\left(1-3 S_{d}^{2}\right) \psi_{1}-\frac{d^{2}}{r^{2}} \psi_{1}-\frac{2 d}{r^{2}} \partial_{\theta} \psi_{2} \\
\hat{L}_{2,2}\left[\psi_{1}, \psi_{2}\right]=\Delta \psi_{2}+\left(1-S_{d}^{2}\right) \psi_{2}-\frac{d^{2}}{r^{2}} \psi_{2}+\frac{2 d}{r^{2}} \partial_{\theta} \psi_{1}
\end{gathered}
$$

Set a function space

$$
\Sigma=\left\{\left(\begin{array}{l}
\phi \\
\psi
\end{array}\right)=\left(\begin{array}{l}
\phi(z) \\
\psi(z)
\end{array}\right) \in \mathbb{R} \times \mathbb{C} \mid \begin{array}{ll}
\phi\left(z e^{\sqrt{-1} \frac{2 \pi}{k}}\right)=\phi(z), & \phi(\bar{z})=\phi(z) \\
\psi\left(z e^{\sqrt{-1} \frac{2 \pi}{k}}\right)=e^{\sqrt{-1} \frac{2 \pi}{k}} \psi(z), & \psi(\bar{z})=\psi(z)^{*}
\end{array}\right\}
$$

where $k \geq 2$ is an integer. Hereafter, both the over-bar and asterisk denote complex conjugate. We remark that the equation (1.5) is invariant under the following two maps

$$
\left\{\begin{array}{l}
T_{1}\left(\begin{array}{c}
\phi \\
\psi
\end{array}\right)=\left(\begin{array}{c}
\phi\left(z e^{\sqrt{-1} \frac{2 \pi}{k}}\right) \\
e^{-\sqrt{-1} \frac{2 \pi}{k}} \psi\left(z e^{\sqrt{-1} \frac{2 \pi}{k}}\right)
\end{array}\right), \\
T_{2}\left(\begin{array}{c}
\phi \\
\psi
\end{array}\right)=\left(\begin{array}{c}
\phi(\bar{z}) \\
\psi(\bar{z})^{*}
\end{array}\right) .
\end{array}\right.
$$

Therefore, we may look for solutions of (1.5) in the space $\sum$. We first have

\section{LEMMA 2.1.}

(1) Suppose $L_{1}[\phi]=0, \phi \in H^{2}\left(\mathbb{R}^{2}\right)$ and $\phi(\bar{z})=\phi(z)$. Then $\phi(z)=c \frac{\partial w}{\partial z_{1}}(z)$, where $z=$ $z_{1}+\sqrt{-1} z_{2}, z_{j} \in \mathbb{R}$ and $c$ is a constant. 
(2) Suppose

$$
L_{2}[\psi]=0,|\psi| \leq C, \psi(z)^{*}=\psi(\bar{z}), \text { and } \psi\left(z e^{\sqrt{-1} \frac{2 \pi}{k}}\right)=e^{\sqrt{-1} \frac{2 \pi}{k}} \psi(z),
$$

where $C$ is a positive constant. Then $\psi \equiv 0$, provided that $|d|=1$ or $|d|>1$ and $2(d-1) \not \equiv 0 \bmod k$.

Proof. (1) is easy to show. See Appendix C of [20]. We only need to show (2). We firstly state the proof for the case when $|d|=1$. For simplicity, we may assume $d=1$. From [21](Theorem 3.2), we know that

$$
\psi=c_{0}(\underbrace{\sqrt{-1} S(r) e^{\sqrt{-1} \theta}}_{\psi_{0,0}})+\sum_{j=1}^{2} c_{j} \underbrace{\frac{\partial}{\partial z_{j}}\left(S(r) e^{\sqrt{-1} \theta}\right)}_{\psi_{0, j}^{\|}},
$$

where $c_{j}$ 's are constants. It is easy to calculate that $\left(\sqrt{-1} S(r) e^{\sqrt{-1} \theta}\right)^{*}=-\sqrt{-1} S(r) e^{-\sqrt{-1} \theta}$, $\psi_{0,1}=\left(\frac{S(r)}{r}\right)^{\prime} \frac{x^{2}}{r}+\frac{S(r)}{r}+\sqrt{-1}\left(\frac{S(r)}{r}\right)^{\prime} x y$, and $\psi_{0,2}=\left(\frac{S(r)}{r}\right)^{\prime} \frac{x y}{r}+\sqrt{-1}\left[\left(\frac{S(r)}{r}\right)^{\prime} y^{2}+\frac{S(r)}{r}\right]$, where $x=z_{1}$ and $y=z_{2}$. Consequently, $\left(\psi_{0,0}(z)\right)^{*}=-\psi_{0,0}(\bar{z}),\left(\psi_{0,1}(z)\right)^{*}=\psi_{0,1}(\bar{z})$ and $\left(\psi_{0,2}(z)\right)^{*} \neq \psi_{0,2}(\bar{z})$. Moreover, due to $\psi(z)^{*}=\psi(\bar{z})$, we have $c_{0}=c_{2}=0$. Hence we only have

$$
\psi(z)=c_{1} \psi_{0,1}(z) \text {. }
$$

However, it is obvious that $\psi_{0,1}$ doesn't satisfy $\psi_{0,1}\left(z e^{\sqrt{-1} \frac{2 \pi}{k}}\right)=e^{\sqrt{-1} \frac{2 \pi}{k}} \psi_{0,1}(z)$. Thus $c_{1}=0$ and $\psi \equiv 0$.

Now we give the proof for the case that $|d|>1$. From [14], the solution $\psi$ locally may become a linear combination of $\psi_{d, 0}(z)=\sqrt{-1} h(r) e^{\sqrt{-1} d \theta}$ and the following forms

$$
\psi_{d, m}(z)=a(r) e^{\sqrt{-1}(d-m) \theta}+b(r) e^{\sqrt{-1}(d+m) \theta},
$$

for $m \in \mathbb{N}$, where $z=r e^{\sqrt{-1} \theta}$ and $(r, \theta)$ is the polar coordinate. Here $h, a$ and $b$ are real-valued functions. Actually, these forms are invariant to the operator $L_{2}$ so one may decompose the function space $\Sigma$ as invariant subspaces having the forms like $\psi_{d, 0}$ and $\psi_{d, m}$ 's. Then the condition $\psi(z)^{*}=\psi(\bar{z})$ may imply $\psi_{d, 0}(z)^{*}=\psi_{d, 0}(\bar{z})$. However, since $\psi_{d, 0}(z)=\sqrt{-1} h(r) e^{\sqrt{-1} d \theta}$, then $\psi_{d, 0}(z)^{*}=-\psi_{d, 0}(\bar{z})$ which gives $\psi_{d, 0}(z) \equiv 0$. Besides, the condition $\psi\left(z e^{\sqrt{-1} \frac{2 \pi}{k}}\right)=e^{\sqrt{-1} \frac{2 \pi}{k}} \psi(z)$ may give $\psi_{d, m}\left(z e^{\sqrt{-1} \frac{2 \pi}{k}}\right)=e^{\sqrt{-1} \frac{2 \pi}{k}} \psi_{d, m}(z)$. Consequently,

$$
a(r) e^{\sqrt{-1}(d-m) \theta}\left(e^{\sqrt{-1}(d-m) \frac{2 \pi}{k}}-e^{\sqrt{-1} \frac{2 \pi}{k}}\right)+b(r) e^{\sqrt{-1}(d+m) \theta}\left(e^{\sqrt{-1}(d+m) \frac{2 \pi}{k}}-e^{\sqrt{-1} \frac{2 \pi}{k}}\right)=0 .
$$

Hence $a \equiv 0$ or $b \equiv 0$ if $e^{\sqrt{-1}(d-m-1) \frac{2 \pi}{k}} \neq 1$ or $e^{\sqrt{-1}(d+m-1) \frac{2 \pi}{k}} \neq 1$. Due to $L_{2}\left[\psi_{d, m}\right]=0, a(r)$ and $b(r)$ satisfy

$$
\left\{\begin{array}{l}
a^{\prime \prime}+\frac{1}{r} a^{\prime}-\frac{(d-m)^{2}}{r^{2}} a+\left(1-S_{d}^{2}\right) a-(a+b) S_{d}^{2}=0 \\
b^{\prime \prime}+\frac{1}{r} b^{\prime}-\frac{(d+m)^{2}}{r^{2}} b+\left(1-S_{d}^{2}\right) b-(a+b) S_{d}^{2}=0
\end{array}\right.
$$


This implies $a \equiv b \equiv 0$ if $a \equiv 0$ or $b \equiv 0$. Thus $a \equiv b \equiv 0$ if $e^{\sqrt{-1}(d-m-1) \frac{2 \pi}{k}} \neq 1$ or $e^{\sqrt{-1}(d+m-1) \frac{2 \pi}{k}} \neq 1$. It is trivial that $e^{\sqrt{-1}(d-m-1) \frac{2 \pi}{k}} \neq 1$ or $e^{\sqrt{-1}(d+m-1) \frac{2 \pi}{k}} \neq 1$ if $2(d-1) \not \equiv 0$ $\bmod k$. Therefore $a \equiv b \equiv 0$ i.e. $\psi_{d, m} \equiv 0$ if $2(d-1) \not \equiv 0 \bmod k$, and we may complete the proof of Lemma 2.1.

To study the properties of $L_{2}$ (or $\hat{L}_{2}$ ), we introduce some Sobolev spaces. Let $\alpha \in\left(0, \frac{1}{2}\right)$. We introduce Hilbert spaces $X_{\alpha}$ and $Y_{\alpha}$ as follows:

$$
X_{\alpha}=\left\{u=u_{1}+\sqrt{-1} u_{2} \in L_{l o c}^{2}\left(\mathbb{R}^{2} ; \mathbb{C}\right) \mid \int_{\mathbb{R}^{2}}\left(1+|x|^{2+\alpha}\right)\left(\left|u_{1}\right|+\left|u_{2}\right|\right)^{2}<+\infty\right\}
$$

equipped with inner product $(u, v)_{X_{\alpha}}=\int_{\mathbb{R}^{2}}\left(1+|x|^{2+\alpha}\right)\left(u_{1} v_{1}+u_{2} v_{2}\right) d x$, and

$$
Y_{\alpha}=\left\{v=v_{1}+\left.\sqrt{-1} v_{2} \in W_{l o c}^{2,2}\left(\mathbb{R}^{2} ; \mathbb{C}\right)\left|\int_{\mathbb{R}^{2}}\right| \triangle v\right|^{2}\left(1+|x|^{2+\alpha}\right) d x+\int_{\mathbb{R}^{2}} \frac{|v|^{2}}{1+|x|^{2+\alpha}} d x<+\infty\right\}
$$

equipped with inner product $(u, v)_{Y_{\alpha}}=(\triangle u, \triangle v)_{X_{\alpha}}+\int_{\mathbb{R}^{2}} \frac{u \cdot v}{1+|x|^{2+\alpha}} d x$, respectively. Thanks to the inequality

$$
\int_{\mathbb{R}^{2}}|h| \leq\left(\int_{\mathbb{R}^{2}} \frac{1}{(1+|z|)^{2+\alpha}}\right)^{\frac{1}{2}}\|h\|_{X_{\alpha}} .
$$

Besides, we see that $X_{\alpha}$ has a compact embedding to $L^{1}\left(\mathbb{R}^{2}\right)$. Originally, these spaces are realvalued function spaces introduced in Chae and Imanuvilor (cf. [5]). Here we generalize $X_{\alpha}, Y_{\alpha}$ as complex-valued function spaces, and regard the operator $L_{2}$ from the space $Y_{\alpha}$ to the space $X_{\alpha}$. We list some properties of $X_{\alpha}$ and $Y_{\alpha}$, whose proofs are exactly the same as in [5].

\section{LEMMA 2.2.}

(1) Let $v \in Y_{\alpha}$ be a harmonic function. Then $v=$ constant.

(2) $\forall v \in Y_{\alpha}$, we have $|v(z)| \leq C_{1}\|v\|_{Y_{\alpha}}(\ln (1+|z|)+1), \forall z \in \mathbb{R}^{2}$.

(3) The image of $L_{2}$ (or $\hat{L}_{2}$ ) is closed in $X_{\alpha} \cap \Sigma_{0}$, where $\Sigma_{0} \equiv\{\psi=\psi(z) \in \mathbb{C}:(0, \psi) \in \Sigma\}$.

Now we study the invertibility of $L_{2}$ (or $\hat{L}_{2}$ ) on the space $Y_{\alpha} \cap \Sigma_{0}$.

LEMMA 2.3. For $\alpha \in\left(0, \frac{1}{2}\right.$ ). Then operator $L_{2}$ (or $\hat{L}_{2}$ ) from the space $Y_{\alpha} \cap \Sigma_{0}$ onto the space $X_{\alpha} \cap \Sigma_{0}$ is invertible. Furthermore,

$$
\|\psi\|_{Y_{\alpha}} \leq C\left\|L_{2}[\psi]\right\|_{X_{\alpha}}, \quad\|\psi\|_{Y_{\alpha}} \leq C\left\|\hat{L}_{2}[\psi]\right\|_{X_{\alpha}}
$$

Proof. It suffices to consider the invertibility of $\hat{L}_{2}$. Then the invertibility of $L_{2}$ follows from a trivial transformation. To show the invertibility of $\hat{L}_{2}$, we claim that $\left(\operatorname{Im}\left(\hat{L}_{2}\right)\right)^{\perp}=\{0\}$. Suppose $\psi \in\left(\operatorname{Im}\left(\hat{L}_{2}\right)\right)^{\perp}$. Then it is easy to check that $\hat{L}_{2}[\psi]=0, \psi \in X_{\alpha} \cap \Sigma_{0}$. By Lemma 2.1. we just need to show that $\psi$ is bounded. To this end, we note that since $\psi \in \Sigma_{0}$, we have 
$\psi(0)=e^{\sqrt{-1} \frac{2 \pi}{k}} \psi(0)$ and $k \geq 2$ which may give $\psi(0)=0$. Let $\phi=\psi e^{\sqrt{-1} d \theta}$. Then $\phi \in X_{\alpha}$ and $L_{2}[\phi]=0$. Hence $\phi \in Y_{\alpha}$ and

$$
\triangle \phi(z)=h(z) /\left(1+|z|^{2+\alpha}\right)^{1 / 2}, \forall z \in \mathbb{R}^{2},
$$

where $h \in L^{2}\left(\mathbb{R}^{2}\right)$. Consequently, by Lemma 2.2 and Riesz representation formula, we may obtain

$$
|\phi(z)| \leq C \log (1+|z|), \quad \forall z \in \mathbb{R}^{2}
$$

and

$$
\left|\partial_{\theta} \phi(z)\right| \leq C \log (1+|z|), \quad \forall z \in \mathbb{R}^{2}
$$

Thus

$$
|\psi(z)|,\left|\partial_{\theta} \psi(z)\right| \leq C \log (1+|z|), \quad \forall z \in \mathbb{R}^{2} .
$$

The equation $\hat{L}_{2}[\psi]=0, \psi=\psi_{1}+\sqrt{-1} \psi_{2}$ can be written as

$$
\begin{aligned}
\Delta \psi_{1}+\left(1-3 S_{d}^{2}\right) \psi_{1}-\frac{d^{2}}{r^{2}} \psi_{1}-\frac{2 d}{r^{2}} \partial_{\theta} \psi_{2} & =0 \\
\Delta \psi_{2}+\left(1-S_{d}^{2}\right) \psi_{2}-\frac{d^{2}}{r^{2}} \psi_{2}+\frac{2 d}{r^{2}} \partial_{\theta} \psi_{1} & =0 .
\end{aligned}
$$

From (2.12) and (2.13), $\psi_{1}$ satisfies

$$
\left|\Delta \psi_{1}+\left(1-3 S_{d}^{2}\right) \psi_{1}-\frac{d^{2}}{r^{2}} \psi_{1}\right| \leq \frac{C}{r^{2}} \log r \quad \text { for } r=|z|>1
$$

Hence by comparison principle,

$$
\left|\psi_{1}(z)\right| \leq \frac{C}{1+|z|}
$$

Here we have used the fact that $S_{d}(r) \sim 1$ as $r \rightarrow \infty$. Similarly, we may have

$$
\left|\partial_{\theta} \psi_{1}(z)\right| \leq \frac{C}{1+|z|}
$$

It remains to show that $\psi_{2}$ is bounded. Now we can use (2.14) and (2.16) to get

$$
\left|\Delta \psi_{2}+\left(1-S_{d}^{2}\right) \psi_{2}-\frac{d^{2}}{r^{2}} \psi_{2}\right| \leq \frac{C}{r^{2}(1+r)} \quad \text { for } r=|z|>1 .
$$

In fact, $\psi_{2}$ satisfies

$$
-\Delta \psi_{2}=f(z), \text { where } f(z)=\left(1-S_{d}^{2}-\frac{d^{2}}{r^{2}}\right) \psi_{2}+\frac{2 d}{r^{2}} \partial_{\theta} \psi_{1}
$$

Since $\psi \in \Sigma_{0}$, we deduce that $f(z) \in X_{\alpha} \cap \Sigma_{0}$ and $\int_{\mathbb{R}^{2}} f(z)=0$. Since $\psi_{2}(0)=0$, we have

$$
\psi_{2}(z)=\psi_{2}(0)+\frac{1}{2 \pi} \int_{\mathbb{R}^{2}} \log \frac{|\tau|}{|z-\tau|} f(\tau) d \tau=\frac{1}{2 \pi} \int_{\mathbb{R}^{2}} \log \frac{|\tau||z|}{|z-\tau|} f(\tau) d \tau
$$

from which we obtain that

$$
\left|\psi_{2}(z)\right| \leq C\|f\|_{X_{\alpha}}<+\infty \quad \text { for } z \in \mathbb{R}^{2} .
$$

Therefore by (2.15) and (2.18), we may complete the proof of Lemma 2.3 . 
To study the vortex solutions, we perform the following key transformation:

$$
v=v_{d}(z) e^{\sqrt{-1} \psi(z)}, \quad v_{d}(z)=S_{d}(r) e^{\sqrt{-1} d \theta},
$$

where $\psi=\psi_{1}+\sqrt{-1} \psi_{2}$ and $\psi_{j} \in \mathbb{R}, j=1,2$. (Here we have assumed that the vortex occurs at the center.) Now we define

$$
\begin{gathered}
S_{1}[u, \psi]:=\Delta u-u+u^{3}+\beta u S_{d}^{2} e^{-2 \psi_{2}}, \\
S_{2}[u, \psi]:=\Delta \psi+\frac{2 \nabla v_{d}}{v_{d}} \cdot \nabla \psi-\sqrt{-1} S_{d}^{2}\left(1-e^{-2 \psi_{2}}\right)+\sqrt{-1}|\nabla \psi|^{2}-\sqrt{-1} \beta u^{2} .
\end{gathered}
$$

We also write

$$
S_{2}[u, \psi]=S_{2}[u, 0]+\tilde{L}_{2}[\psi]+N[u, \psi]
$$

where

$$
\begin{aligned}
\tilde{L}_{2}[\psi]= & \Delta \psi+\frac{2 \nabla v_{d}}{v_{d}} \nabla \psi-2 \sqrt{-1} S_{d}^{2} \psi_{2} \\
= & {\left[\Delta \psi_{1}+2\left(\frac{S_{d}^{\prime}(|z|)}{S_{d}(|z|)} \frac{z}{|z|}\right) \nabla \psi_{1}-d \nabla \theta \cdot \nabla \psi_{2}\right] } \\
& \quad+\sqrt{-1}\left[\Delta \psi_{2}-2 S_{d}^{2} \psi_{2}+2\left(\frac{S_{d}^{\prime}}{S_{d}} \frac{z}{|z|}\right) \nabla \psi_{2}+d \nabla \theta \cdot \nabla \psi_{1}\right], \\
& N[u, \psi]=\sqrt{-1}|\nabla \psi|^{2}-\sqrt{-1} S_{d}^{2}\left(1-e^{-2 \psi_{2}}-2 \psi_{2}\right),
\end{aligned}
$$

where $\nabla \theta=\frac{1}{r}(-\sin \theta, \cos \theta)$. Then it is easy to see that solving (1.5) is equivalent to solving

$$
S_{1}[u, \psi]=0, \quad S_{2}[u, \psi]=0 .
$$

Let $\psi=\psi_{1}+\sqrt{-1} \psi_{2}, h=h_{1}+\sqrt{-1} h_{2}$. We may define two norms as follows:

$$
\|\psi\|_{*}=\sup _{z \in \mathbb{R}^{2}}\left[\left|\psi_{1}\right|+(1+|z|)\left|\nabla \psi_{1}\right|+(1+|z|)^{1+\alpha}\left|\psi_{2}\right|+(1+|z|)^{1+\alpha}\left|\nabla \psi_{2}\right|\right],
$$

and

$$
\|h\|_{* *}=\sup _{z \in \mathbb{R}^{2}}\left[(1+|z|)^{2+\alpha}\left(\left|h_{1}\right|+\left|h_{2}\right|\right)\right] .
$$

Then we may show the following key lemma

LEMMA 2.4. For any $h \in X_{\alpha} \cap \Sigma_{0}$ with $\|h\|_{* *}<+\infty$, there exists a unique $\tilde{\psi} \in Y_{\alpha} \cap \Sigma_{0}$ such that

$$
\tilde{L}_{2}[\psi]=h
$$

Furthermore, we have

$$
\|\psi\|_{*} \leq C\|h\|_{* *}
$$


Proof: Let $\hat{h}=-\sqrt{-1} v_{d} h$ and $\hat{\phi}=-\sqrt{-1} v_{d} \psi$. Then (2.25) is equivalent to

$$
L_{2}[\hat{\phi}]=\hat{h}
$$

where $\hat{h}$ satisfies $\hat{h} \in \Sigma_{0}$ and

$$
\|\hat{h}\|_{* *} \leq\|h\|_{* *}<+\infty .
$$

Consequently, (2.28) implies that $\int_{\mathbb{R}^{2}}|\hat{h}|^{2}\left(1+|z|^{2+\alpha}\right)<+\infty$ and hence $\hat{h} \in X_{\alpha} \cap \Sigma_{0}$. By Lemma 2.3, (2.27) has a unique solution $\phi$. Furthermore, as for (2.17), we obtain

$$
\hat{\phi}(z)=\frac{1}{2 \pi} \int_{\mathbb{R}^{2}} \log \frac{|\tau||z|}{|z-\tau|} \hat{f}(\tau) d \tau
$$

where $|\hat{f}(z)|(1+|z|)^{2+\alpha} \leq C\|h\|_{* *}$. From (2.29), we may deduce that $|\hat{\phi}|+(1+|z|)|\nabla \hat{\phi}| \leq C\|h\|_{* *}$ which implies that $|\psi|+(1+|z|)|\nabla \psi| \leq C\|h\|_{* *}$ i.e. $\left|\psi_{j}\right|+(1+|z|)\left|\nabla \psi_{j}\right| \leq C\|h\|_{* *}, j=1,2$.

To obtain better estimates for $\psi_{2}$, we may use the equation for $\psi_{2}$ and (2.22). Then

$$
\Delta \psi_{2}-2 S_{d}^{2} \psi_{2}+O\left(\frac{1}{|z|}|\nabla \psi|\right)=O\left(\|h\|_{* *}(1+|z|)^{-2-\alpha}\right)
$$

which gives

$$
-\frac{C\|h\|_{* *}}{(1+|z|)^{2}} \leq-\Delta \psi_{2}+2 S_{d}^{2} \psi_{2} \leq \frac{C\|h\|_{* *}}{(1+|z|)^{2}} .
$$

Hence we may use a barrier and elliptic estimates to get

$$
\left|\psi_{2}\right|+\left|\nabla \psi_{2}\right| \leq \frac{C\|h\|_{* *}}{(1+|z|)^{1+\alpha}} .
$$

Here we have used the fact that $S_{d}(r) \sim 1$ as $r \rightarrow \infty$. Therefore we may complete the proof of Lemma 2.4.

\section{Approximate Solutions and Error Estimates}

In this section, we introduce some approximate solutions and derive some useful estimates. Let

$$
S[u, \psi]=\left(\begin{array}{c}
S_{1}[u, \psi] \\
S_{2}[u, \psi]
\end{array}\right) .
$$

Let

$$
\begin{gathered}
\xi_{j}=l e^{\sqrt{-1 \frac{2 \pi}{k}(j-1)}}, j=1, \ldots, k, w_{j}(z):=w\left(z-\xi_{j}\right), \\
u_{l}(z)=\sum_{j=1}^{k} w_{j}(z),
\end{gathered}
$$

Then we have 
LEMMA 3.1. For l large enough, we have

$$
\left\|S_{1}\left[u_{l}, 0\right]\right\|_{L^{2}\left(\mathbb{R}^{2}\right)}+\left\|S_{2}\left[u_{l}, 0\right]\right\|_{* *} \leq C\left(|\beta| l^{2+\alpha}+e^{-2 l \sin \frac{\pi}{k}}\right) .
$$

Proof. It is easy to check that

$$
\begin{aligned}
S_{1}\left[u_{l}, 0\right] & =\triangle u_{l}-u_{l}+u_{l}^{3}+\beta u_{l}\left|v_{d}\right|^{2} \\
& =\left(\sum_{j} w_{j}(z)\right)^{3}-\sum_{j} w_{j}^{3}+\beta \sum_{j} w_{j} S_{d}^{2} \\
& =O\left(\sum_{i \neq j} w_{i}^{2} w_{j}+|\beta| \sum_{j}\left|w_{j}\right|\right) .
\end{aligned}
$$

Due to

$$
\int_{\mathbb{R}^{2}} w_{i}^{4} w_{j}^{2} \leq C e^{-2\left|\xi_{i}-\xi_{j}\right|} \leq C e^{-4 l \sin \frac{\pi}{k}}, \quad \forall i \neq j
$$

we may obtain

$$
\left\|S_{1}\left[u_{l}, 0\right]\right\|_{L^{2}} \leq\left[e^{-2 l \sin \frac{\pi}{k}}+|\beta|\right] .
$$

Here we have used the fact that $\xi_{j}$ 's are vertices of regular $k$-polygon with side length $2 l \sin \frac{\pi}{k}$. On the other hand, we have

$$
\left|S_{2}\left[u_{l}, 0\right]\right|=|\beta| u_{l}^{2} \leq C \sum_{j=1}^{k}|\beta| e^{-2\left|z-\xi_{j}\right|}
$$

and so by (1.21)

$$
\left\|S_{2}\left[u_{l}, 0\right]\right\|_{* *} \leq C|\beta| \sup _{z \in \mathbb{R}^{2}}\left(\sum_{j=1}^{k}|z|^{2+\alpha} e^{-2\left|z-\xi_{j}\right|}\right) \leq C|\beta| \sum_{j=1}^{k}\left|\xi_{j}\right|^{2+\alpha} \leq C|\beta||l|^{2+\alpha} .
$$

Therefore by (3.3) and (3.4), we may obtain (3.1) and complete the proof of Lemma 3.1 .

\section{Liapunov-Schmidt reduction process}

Let $\widetilde{X}:=\left(L^{2}\left(\mathbb{R}^{2}\right) \times X_{\alpha}\right) \cap \Sigma, \widetilde{Y}:=\left(H^{2}\left(\mathbb{R}^{2}\right) \times Y_{\alpha}\right) \cap \Sigma$ and $L:=\left(\begin{array}{c}\widetilde{L}_{1} \\ \widetilde{L}_{2}\end{array}\right): \widetilde{Y} \rightarrow \widetilde{X}$, where $\widetilde{L}_{1}[\phi] \equiv \triangle \phi-\phi+3 u_{l}^{2} \phi$ and $\widetilde{L}_{2}$ is defined by (2.21). To solve (2.23), we first consider the following linear problem: Given $f \in L^{2}\left(\mathbb{R}^{2}\right) \cap \Sigma_{1}$, find $(\phi, c)$ such that

$$
\left\{\begin{array}{l}
\widetilde{L}_{1} \phi=f+c \frac{\partial u_{l}}{\partial l}, \quad \phi \in H^{2}\left(\mathbb{R}^{2}\right) \cap \Sigma_{1} \\
\int_{\mathbb{R}^{2}} \phi \frac{\partial u_{l}}{\partial l}=0
\end{array}\right.
$$

where $\Sigma_{1} \equiv\{\phi=\phi(z) \in \mathbb{R}:(\phi, 0) \in \Sigma\}$. 
LEMMA 4.1. For each $f \in L^{2}\left(\mathbb{R}^{2}\right) \cap \Sigma_{1}$, there exists a unique pair $(\phi, c)$ satisfying 4.1 ) such that

$$
\|\phi\|_{H^{2}} \leq C\|f\|_{L^{2}} .
$$

PROOF. The existence and uniqueness may follow from Fredholm's alternatives. Now we prove (4.2) by contradiction. Suppose (4.2) is not true. Then there exist $\beta_{n}, l_{n} \in \mathbb{R}, \phi_{n} \in$ $H^{2}\left(\mathbb{R}^{2}\right) \cap \Sigma_{1}, f_{n} \in L^{2}\left(\mathbb{R}^{2}\right) \cap \Sigma_{1}$ and $c_{n}$ such that $\beta_{n} \rightarrow 0, l_{n} \rightarrow+\infty$,

$$
\left\|f_{n}\right\|_{L^{2}} \rightarrow 0, \quad\left\|\phi_{n}\right\|_{H^{2}}=1
$$

as $n \rightarrow \infty$,

$$
\widetilde{L}_{1} \phi_{n}=f_{n}+c_{n} \frac{\partial u_{l}}{\partial l}
$$

and

$$
\int_{\mathbb{R}^{2}} \phi_{n} \frac{\partial u_{l}}{\partial l}=0 .
$$

Let $\widetilde{\phi}_{n}(z):=\phi_{n}\left(z+\xi_{1}\right)$. Then by (4.4), $\widetilde{\phi}_{n}$ satisfies

$$
\begin{aligned}
& \triangle \widetilde{\phi}_{n}(z)-\widetilde{\phi}_{n}(z)+3 w^{2}(z) \widetilde{\phi}_{n}(z) \\
& +3\left[2 \sum_{j=2}^{k} w(z) w\left(z+\xi_{1}-\xi_{j}\right)+\left(\sum_{j=2}^{k} w\left(z+\xi_{1}-\xi_{j}\right)\right)^{2}\right] \widetilde{\phi}_{n}(z) \\
& =f_{n}\left(z+\xi_{1}\right)+c_{n}\left[-\frac{\partial w}{\partial z_{1}}(z)+\sum_{j=2}^{k} \frac{\partial w_{j}}{\partial l}\left(z+\xi_{1}\right)\right] .
\end{aligned}
$$

We may multiply (4.4) by $\frac{\partial u_{l}}{\partial l}$ and integrate over the whole space $\mathbb{R}^{2}$. Then by (4.3), it is obvious that

$$
\begin{aligned}
c_{n} \int_{\mathbb{R}^{2}}\left(\frac{\partial u_{l}}{\partial l}\right)^{2} & =\int_{\mathbb{R}^{2}}\left(\triangle \phi_{n}-\phi_{n}+3 u_{l}^{2} \phi_{n}\right) \frac{\partial u_{l}}{\partial l}-\int_{\mathbb{R}^{2}} f_{n} \frac{\partial u_{l}}{\partial l} \\
& =\int_{\mathbb{R}^{2}}\left(\triangle \frac{\partial u_{l}}{\partial l}-\frac{\partial u_{l}}{\partial l}+3 u_{l}^{2} \frac{\partial u_{l}}{\partial l}\right) \phi_{n}-\int_{\mathbb{R}^{2}} f_{n} \frac{\partial u_{l}}{\partial l} \\
& \stackrel{n \rightarrow+\infty}{\longrightarrow} 0 .
\end{aligned}
$$

So $c_{n} \rightarrow 0$ as $n \rightarrow+\infty$. Hence by (4.6) , as $n \rightarrow \infty, \widetilde{\phi}_{n} \rightarrow \widetilde{\phi}_{0}$ which satisfies $\triangle \widetilde{\phi}_{0}-\widetilde{\phi}_{0}+3 w^{2} \widetilde{\phi}_{0}=0$ in $\mathbb{R}^{2}$. Thus by [20], $\widetilde{\phi}_{0}=\sum_{j=1}^{2} a_{j} \frac{\partial w}{\partial z_{j}}$ for some constants $a_{1}$ and $a_{2}$. Moreover, due to $\widetilde{\phi}_{n}(\bar{z})=$ $\widetilde{\phi}_{n}(z)$, we have $\widetilde{\phi}_{0}(\bar{z})=\widetilde{\phi}_{0}(z)$. Consequently, $a_{2}=0$ and $\widetilde{\phi}_{0}=a_{1} \frac{\partial w}{\partial z_{1}}$. On the other hand, by 
(4.5),

$$
\begin{aligned}
0 & =\int_{\mathbb{R}^{2}} \phi_{n} \frac{\partial u_{l}}{\partial l} \\
& =\int_{\mathbb{R}^{2}} \widetilde{\phi}_{n}(z)\left[-\frac{\partial w}{\partial z_{1}}(z)+\sum_{j=2}^{k} \frac{\partial w_{j}}{\partial l}\left(z+\xi_{1}\right)\right] \\
& \stackrel{n \rightarrow+\infty}{\longrightarrow}-a_{1} \int_{\mathbb{R}^{2}}\left(\frac{\partial w}{\partial z_{1}}\right)^{2} .
\end{aligned}
$$

Therefore $a_{1}=0, \widetilde{\phi}_{0}=0$, and $\widetilde{\phi}_{n} \rightarrow 0$ in $L_{l o c}^{2}\left(\mathbb{R}^{2}\right)$. Then we have $3 u_{l}^{2} \widetilde{\phi}_{n} \rightarrow 0$ in $L^{2}$ and hence $\left\|\phi_{n}\right\|_{H^{2}} \rightarrow 0$ which may give a contradiction and complete the proof.

From Lemma 2.4 and 4.1, we may obtain that

LEMMA 4.2. For $\left(\begin{array}{c}f_{1} \\ f_{2}\end{array}\right) \in \widetilde{X}$, there exists a unique $\left(\left(\begin{array}{c}\phi \\ \psi\end{array}\right), c\right) \in \widetilde{Y} \times \mathbb{R}$, such that

$$
\widetilde{L}\left(\begin{array}{c}
\phi \\
\psi
\end{array}\right)=\left(\begin{array}{c}
f_{1} \\
f_{2}
\end{array}\right)+c\left(\begin{array}{c}
\frac{\partial u_{l}}{\partial l} \\
0
\end{array}\right), \quad \int_{\mathbb{R}^{2}} \phi \frac{\partial u_{l}}{\partial l}=0 .
$$

Moreover, we have $\|\phi\|_{H^{2}}+\|\psi\|_{*} \leq C\left(\left\|f_{1}\right\|_{L^{2}}+\left\|f_{2}\right\|_{* *}\right)$.

We may denote $A$ as the inverse operator for Lemma 4.2, i.e. $A\left(\begin{array}{c}f_{1} \\ f_{2}\end{array}\right)=\left(\begin{array}{l}\phi \\ \psi\end{array}\right)$. Finally, we have

LEMMA 4.3. For $l$ large satisfying (1.21), there exists a unique $\left(\begin{array}{c}\phi_{l} \\ \psi_{l}\end{array}\right) \in \widetilde{Y}$ such that

$$
S\left[u_{l}+\phi_{l}, \psi_{l}\right]=c(l)\left(\begin{array}{c}
\frac{\partial u_{l}}{\partial l} \\
0
\end{array}\right), \quad \int_{\mathbb{R}^{2}} \phi_{l} \frac{\partial u_{l}}{\partial l}=0 .
$$

Furthermore,

$$
\left\|\phi_{l}\right\|_{H^{2}}+\left\|\psi_{l}\right\|_{*} \leq C\left(e^{-2 l \sin \frac{\pi}{k}}+|\beta| l^{2+\alpha}\right) .
$$

PROOF. This may follow from standard contraction mapping principle. We choose $(\phi, \psi) \in$ $B$, where

$$
B=\left\{(\phi, \psi) \in \widetilde{Y}:\|\phi\|_{H^{2}}+\|\psi\|_{*} \leq C\left(e^{-2 l \sin \frac{\pi}{k}}+|\beta| l^{2+\alpha}\right)\right\},
$$

and then expand

$$
\begin{aligned}
S_{1}\left[u_{l}+\phi, \psi\right] & =S_{1}\left[u_{l}, 0\right]+\tilde{L}_{1}[\phi]+N_{1}[\phi, \psi], \\
S_{2}\left[u_{l}+\phi, \psi\right] & =S_{2}\left[u_{l}, 0\right]+\tilde{L}_{2}[\psi]+N_{2}[\phi, \psi],
\end{aligned}
$$


where

$$
N_{1}[\phi, \psi]=3 u_{l} \phi^{2}+\phi^{3}+\beta\left[\phi S_{d}^{2} e^{-2 \psi_{2}}+u_{l} S_{d}^{2}\left(e^{-2 \psi_{2}}-1\right)\right],
$$

and

$$
N_{2}[\phi, \psi]=-\sqrt{-1} \beta\left(2 u_{l} \phi+\phi^{2}\right)+\sqrt{-1}|\nabla \psi|^{2}-\sqrt{-1} S_{d}^{2}\left(1-e^{-2 \psi_{2}}-2 \psi_{2}\right) .
$$

By (1.21) and $(\phi, \psi) \in B$, we have

$$
\|\phi\|_{H^{2}}+\|\psi\|_{*} \leq|\beta|^{1-\sigma}, \quad \sigma \in\left(0, \frac{1}{100}\right)
$$

as $\beta>0$ sufficiently small i.e. $l$ sufficiently large. Moreover, by (4.9), (4.10) and the norms defined at (2.24), we see that

$$
\left\|N_{1}\right\|_{L^{2}\left(\mathbb{R}^{2}\right)} \leq C|\beta|\|\phi\|_{L^{2}}+\|\phi\|_{L^{2}}^{2}+|\beta|
$$

and

$$
\left\|N_{2}\right\|_{* *} \leq C\|\psi\|_{*}^{2}+C|\beta|
$$

Now we can write (4.8) as

$$
\left(\begin{array}{c}
\phi \\
\psi
\end{array}\right)=A\left(\begin{array}{c}
S_{1}\left[u_{l}, 0\right]+N_{1}[\phi, \psi] \\
S_{2}\left[u_{l}, 0\right]+N_{2}[\phi, \psi]
\end{array}\right) .
$$

Then as for the proof of Proposition 1 in [16], we may use a contraction mapping argument to obtain the desired result. Here we also need to use (4.11), (4.12), Lemma 3.1, 4.1 and 4.2.

\section{$5 \quad$ Finding zero of $c(l)$}

To prove Theorem 1.1, it is enough to find a zero of $c(l)$ in (4.8). We multiply the first equation of (4.8) by $\frac{\partial u_{l}}{\partial l}$ and integrate it over the whole space $\mathbb{R}^{2}$. Then we obtain

$$
\begin{aligned}
c(l) \int_{\mathbb{R}^{2}}\left(\frac{\partial u_{l}}{\partial l}\right)^{2}= & \int_{\mathbb{R}^{2}}\left[\triangle\left(u_{l}+\phi_{l}\right)-\left(u_{l}+\phi_{l}\right)+\left(u_{l}+\phi_{l}\right)^{3}\right] \frac{\partial u_{l}}{\partial l} \\
& +\beta \int_{\mathbb{R}^{2}}\left(u_{l}+\phi_{l}\right)\left|v_{d}+\psi_{l}\right|^{2} \frac{\partial u_{l}}{\partial l} \\
= & : I_{1}+I_{2},
\end{aligned}
$$


where

$$
\begin{aligned}
I_{2} & =\beta \int_{\mathbb{R}^{2}} u_{l}\left|v_{d}\right|^{2} \frac{\partial u_{l}}{\partial l}+\beta \int_{\mathbb{R}^{2}} O\left(\left|\phi_{l}\right|+\left|u_{l}+\phi_{l}\right|\left|\psi_{l}\right|\right)\left|\frac{\partial u_{l}}{\partial l}\right| \\
& =\beta \int_{\mathbb{R}^{2}} u_{l} S_{d}^{2} \frac{\partial u_{l}}{\partial l}+O\left(|\beta|^{2} l^{2+\alpha}+|\beta| e^{-2 l \sin \frac{\pi}{k}}\right) \\
& =\beta \int_{\mathbb{R}^{2}} u_{l}\left(1-\frac{d^{2}}{2 r^{2}}+O\left(\frac{1}{r^{4}}\right)\right)^{2} \frac{\partial u_{l}}{\partial l}+O\left(|\beta|^{2} l^{2+\alpha}+|\beta| e^{-2 l \sin \frac{\pi}{k}}\right) \\
& =\beta \int_{\mathbb{R}^{2}} u_{l} \frac{\partial u_{l}}{\partial l}+O\left(|\beta| l^{-4}+|\beta|^{2} l^{2+\alpha}+|\beta| e^{-2 l \sin \frac{\pi}{k}}\right)-\beta \int_{\mathbb{R}^{2}} u_{l} \frac{d^{2}}{r^{2}} \frac{\partial u_{l}}{\partial l} .
\end{aligned}
$$

Note that

$$
\begin{aligned}
\int_{\mathbb{R}^{2}} u_{l} \frac{\partial u_{l}}{\partial l} & =\int_{\mathbb{R}^{2}} \sum_{j=1}^{k} w_{j} \sum_{i \neq j} \frac{\partial w_{i}}{\partial l} \\
& =O\left(e^{-2 l \sin \frac{\pi}{k}}\right), \\
-\beta \int_{\mathbb{R}^{2}} u_{l} \frac{d^{2}}{r^{2}} \frac{\partial u_{l}}{\partial l} & =\beta\left[\int_{\mathbb{R}^{2}} w \frac{d^{2}}{\left|z+\xi_{1}\right|^{2}} \frac{\partial w}{\partial z_{1}}+O\left(e^{-2 l \sin \frac{\pi}{k}}\right)\right] \\
& =-c_{2} \beta l^{-3} \int_{\mathbb{R}^{2}} w^{2}+O\left(|\beta| l^{-5}+|\beta| e^{-2 l \sin \frac{\pi}{k}}\right),
\end{aligned}
$$

where $c_{2}$ is a positive constant independent of $\beta$ and $l$. So

$$
I_{2}=-c_{2} \beta l^{-3} \int_{\mathbb{R}^{2}} w^{2}+O\left(|\beta| l^{-4}+|\beta|^{2} l^{2+\alpha}+|\beta| e^{-2 l \sin \frac{\pi}{k}}\right) .
$$

For $I_{1}$, we have

$$
\begin{aligned}
I_{1} & =\int_{\mathbb{R}^{2}}\left(\triangle u_{l}-u_{l}+u_{l}^{3}\right) \frac{\partial u_{l}}{\partial l}+\int_{\mathbb{R}^{2}}\left(\triangle \phi_{l}-\phi_{l}+3 u_{l}^{2} \phi_{l}\right) \frac{\partial u_{l}}{\partial l}+O\left(|\beta|^{2} l^{2+\alpha}+|\beta| e^{-2 l \sin \frac{\pi}{k}}\right) \\
& =\int_{\mathbb{R}^{2}}\left[\left(\sum_{j} w_{j}\right)^{3}-\sum_{j} w_{j}^{3}\right] \frac{\partial u_{l}}{\partial l}+\int_{\mathbb{R}^{2}}\left[\triangle \frac{\partial u_{l}}{\partial l}-\frac{\partial u_{l}}{\partial l}+3 u_{l}^{2} \frac{\partial u_{l}}{\partial l}\right] \phi_{l}+O\left(|\beta|^{2} l^{2+\alpha}+|\beta| e^{-2 l \sin \frac{\pi}{k}}\right) \\
& =6 k \int_{\mathbb{R}^{2}} w_{1}^{2} w_{2}\left(-\frac{\partial w_{1}}{\partial z_{1}}\right)+O\left(|\beta|^{2} l^{2+\alpha}+|\beta| e^{-2 l \sin \frac{\pi}{k}}\right) \\
& =-6 k \int_{\mathbb{R}^{2}} w^{2}\left(\left|z-\xi_{1}\right|\right) w^{\prime}\left(\left|z-\xi_{1}\right|\right) w\left(\left|z-\xi_{2}\right|\right) \frac{z_{1}-l}{\left|z-\xi_{1}\right|} d z+O\left(|\beta|^{2} l^{2+\alpha}+|\beta| e^{-2 l \sin \frac{\pi}{k}}\right) \\
& =-6 k \int_{\mathbb{R}^{2}} w^{2}(|z|) w^{\prime}(|z|) w\left(\left|z+\xi_{1}-\xi_{2}\right|\right) \frac{z_{1}}{|z|} d z+O\left(|\beta|^{2} l^{2+\alpha}+|\beta| e^{-2 l \sin \frac{\pi}{k}}\right) \\
& =c_{0} \cdot w\left(\left|\xi_{1}-\xi_{2}\right|\right)+O\left(|\beta|^{2} l^{2+\alpha}+|\beta| e^{-2 l \sin \frac{\pi}{k}}\right) \\
& =c_{0}\left(2 \sin \frac{\pi}{k}\right)^{-1 / 2} \cdot l^{-1 / 2} e^{-2 l \sin \frac{\pi}{k}}+O\left(|\beta|^{2} l^{2+\alpha}+|\beta| e^{-2 l \sin \frac{\pi}{k}}\right)
\end{aligned}
$$


where $c_{0}$ is a positive constant. Here we have used the fact that (1.7) holds.

Combining (5.5) and (5.6), we see that

$$
c(l) \approx c_{1} l^{-1 / 2} e^{-2 l \sin \frac{\pi}{k}}-c_{2} \beta l^{-3}+O\left(\beta l^{-5}\right) .
$$

Moreover, we may choose $\hat{l}_{\beta}$ such that

$$
\hat{l}_{\beta}^{-1 / 2} e^{-2 \hat{l}_{\beta} \sin \frac{\pi}{k}}=\beta \hat{l}_{\beta}^{-3} .
$$

Then

$$
\hat{l}_{\beta}=\frac{1}{2 \sin \frac{\pi}{k}} \log \frac{1}{\beta}+c_{3} \log \log \frac{1}{\beta}+c_{4} .
$$

Now we want to choose $l$ such that $l \in\left(\hat{l}_{\beta}-\gamma, \hat{l}_{\beta}+\gamma\right)$ and $c(l)=0$ for some suitable $\gamma>0$. It is remarkable that

$$
c\left(\hat{l}_{\beta}-\gamma\right) \approx c_{1}\left(\hat{l}_{\beta}-\gamma\right)^{-1 / 2} e^{-2 \hat{l}_{\beta} \sin \frac{\pi}{k}+2 \gamma \sin \frac{\pi}{k}}-c_{2} \beta\left(\hat{l}_{\beta}-\gamma\right)^{-3}+O\left(\beta\left(\log \frac{1}{\beta}\right)^{-5}\right)>0,
$$

if $\gamma$ is large enough. Similarly, $c\left(\hat{l}_{\beta}+\gamma\right)<0$ if $\gamma$ is large enough. Since $c(l)$ is continuous in $l$, then by the mean-value theorem, there exists $l_{\beta} \in\left(\hat{l}_{\beta}-\gamma, \hat{l}_{\beta}+\gamma\right)$ such that $c\left(l_{\beta}\right)=0$. Consequently, the function $\left(\begin{array}{c}u_{l_{\beta}}+\phi_{l_{\beta}} \\ v_{d} e^{\sqrt{-1} \psi_{l_{\beta}}}\end{array}\right)=:\left(\begin{array}{c}u_{\beta} \\ v_{\beta}\end{array}\right)$ is a solution of (1.5)). Furthermore, it is easy to check that $\left(u_{\beta}, v_{\beta}\right)$ satisfies all the properties of Theorem 1.1. Therefore we may complete the proof of Theorem 1.1.

\section{Proof of Theorem 1.2}

We first consider problem (1.16) on a ball $B_{R}$ :

$$
\left\{\begin{array}{l}
\Delta u-u+u^{3}+\beta u S^{2}=0, u=u(r), r<R \\
\Delta S-\frac{d^{2}}{r^{2}} S+S\left(1-S^{2}\right)+\beta u^{2} S=0, S=S(r), r<R \\
S(0)=0, S(R)=1, u(R)=0, u>0,0<S<1
\end{array}\right.
$$

Our idea is to find a solution of (6.1), and then let $R \rightarrow+\infty$. To this end, we consider the associated energy functional

$$
\begin{aligned}
E_{R}[u, S]= & \frac{1}{2} \int_{B_{R}}\left(|\nabla u|^{2}+u^{2}\right)+\frac{1}{2} \int_{B_{R}}\left(|\nabla S|^{2}+\frac{d^{2}}{r^{2}} S^{2}\right) \\
& +\frac{1}{4} \int_{B_{R}}\left(1-S^{2}\right)^{2}-\frac{\beta}{2} \int_{B_{R}} S^{2} u^{2}-\frac{1}{4} \int_{B_{R}} u^{4},
\end{aligned}
$$

for $u \in H_{0}^{1}\left(B_{R}\right)$ and $S \in I_{R} \equiv\left\{S \in H^{1}\left(B_{R}\right): S(z)=S(|z|), S(0)=0, S(R)=1\right\}$.

Let the Nehari manifold be

$$
\mathcal{N}=\left\{(u, S) \in H_{0}^{1}\left(B_{R}\right) \times I_{R}, u \geq 0, u \neq \equiv: \int_{B_{R}}\left(|\nabla u|^{2}+u^{2}\right)=\int_{B_{R}}\left(u^{4}+\beta u^{2} S^{2}\right)\right\} .
$$


Then we consider the following energy minimization problem

$$
c_{R}=\inf _{(u, S) \in \mathcal{N}} E_{R}[u, S]
$$

and we have

LEMMA 6.1. If $\beta<0$, then $c_{R}$ is obtained by some radially symmetric function $\left(u_{R}, S_{R}\right)$. Furthermore, $u_{R}^{\prime}(r)<0$ and $S_{R}^{\prime}(r)>0$ for $r>0$.

Proof: We follow the proof of (1) Theorem 3.3 of [15]. To this end, we define another energy functional

$$
\begin{aligned}
E_{R}^{\prime}[u, S]= & \frac{1}{4} \int_{B_{R}}\left(|\nabla u|^{2}+u^{2}\right)+\frac{1}{2} \int_{B_{R}}\left(|\nabla S|^{2}+\frac{d^{2}}{r^{2}} S^{2}\right) \\
& +\frac{1}{4} \int_{B_{R}}\left(1-S^{2}\right)^{2}-\frac{\beta}{4} \int_{B_{R}} S^{2} u^{2}
\end{aligned}
$$

and another solution manifold

$$
\mathcal{N}^{\prime}=\left\{(u, S) \in H_{0}^{1}\left(B_{R}\right) \times I_{R}, u \geq 0, u \neq \equiv 0: \int_{B_{R}}\left(|\nabla u|^{2}+u^{2}\right) \leq \int_{B_{R}}\left(u^{4}+\beta u^{2} S^{2}\right)\right\} .
$$

We consider another minimization problem:

$$
c_{R}^{\prime}=\inf _{(u, S) \in \mathcal{N}^{\prime}} E_{R}^{\prime}[u, S]
$$

Certainly, we have

$$
c_{R}^{\prime} \leq c_{R}
$$

Let $\left(u_{n}, S_{n}\right)$ be a minimizing sequence of $c_{R}^{\prime}$ on $\mathcal{N}^{\prime}$. Replacing $S_{n}$ by $\min \left(S_{n}, 1\right)$, we may assume that $S_{n} \leq 1$. We may denote $u_{n}^{*}$ and $S_{n}^{*}$ as the Schwartz symmetrization of $u_{n}$ and $S_{n}$, respectively. Then $\left(1-S_{n}\right)^{*}=1-S_{n}^{*}$. By Theorem 3.4 of [10],

$$
\int_{B_{R}}\left(1-S_{n}^{2}\right) u_{n}^{2} \leq \int_{B_{R}}\left(1-S_{n}^{2}\right)^{*}\left(u_{n}^{*}\right)^{2}
$$

and hence due to $\beta<0$,

$$
-\beta \int_{B_{R}}\left(u_{n}^{*}\right)^{2}\left(S_{n}^{*}\right)^{2} \leq-\beta \int_{B_{R}} u_{n}^{2} S_{n}^{2}
$$

On the other hand, we also have

$$
\begin{gathered}
\int_{B_{R}}\left(\frac{1}{2}\left(u_{n}^{*}\right)^{2}+\frac{1}{2} \frac{d^{2}}{r^{2}}\left(S_{n}^{*}\right)^{2}-\frac{1}{4}\left(u_{n}^{*}\right)^{4}\right)=\int_{B_{R}}\left(\frac{1}{2} u_{n}^{2}+\frac{1}{2} \frac{d^{2}}{r^{2}} S_{n}^{2}-\frac{1}{4} u_{n}^{4}\right), \\
\int_{B_{R}}\left(\left|\nabla u_{n}^{*}\right|^{2}+\left|\nabla S_{n}^{*}\right|^{2}\right) \leq \int_{B_{R}}\left(\left|\nabla u_{n}\right|^{2}+\left|\nabla S_{n}\right|^{2}\right) .
\end{gathered}
$$


Hence we obtain

$$
E_{R}^{\prime}\left[u_{n}^{*}, S_{n}^{*}\right] \leq E_{R}^{\prime}\left[u_{n}, S_{n}\right]
$$

and

$$
\int_{B_{R}}\left(\left|\nabla u_{n}^{*}\right|^{2}+\left(u_{n}^{*}\right)^{2}\right) \leq \int_{B_{R}}\left(\left(u_{n}^{*}\right)^{4}+\beta\left(u_{n}^{*}\right)^{2}\left(S_{n}^{*}\right)^{2}\right) .
$$

Thus we may replace $\left(u_{n}, S_{n}\right)$ by its symmetrization $\left(u_{n}^{*}, S_{n}^{*}\right)$. Since $S_{n} \leq 1$ and $H^{1}\left(B_{R}\right)$ is a compact embedding to $L^{4}\left(B_{R}\right)$, we see that $\left(u_{n} S_{n}\right) \rightarrow\left(u_{R}, S_{R}\right)$ weakly in $H^{1}\left(B_{R}\right)$ and strongly in $L^{4}\left(B_{R}\right)$, where $\left(u_{R}, S_{R}\right) \in \mathcal{N}^{\prime}$ attains $c_{R}^{\prime}$. So $c_{R}^{\prime}$ is attained. If $\left(u_{R}, S_{R}\right) \in\left(\mathcal{N}^{\prime}\right)^{\circ}$-the interior of $\mathcal{N}^{\prime}$, then $\left(u_{R}, S_{R}\right)$ is a local minimizer of $E_{R}^{\prime}$ and hence we have

$$
\Delta u_{R}-u_{R}+\beta u_{R} S_{R}=0, \quad \forall 0<r<R, \quad \text { and } u_{R}(R)=0,
$$

which implies $u_{R} \equiv 0$ since $\beta<0$. This is impossible since from (6.9) and Sobolev embedding, we infer that $\int_{B_{R}} u_{R}^{4} \geq C>0$. Therefore $\left(u_{R}, S_{R}\right) \in \partial\left(\mathcal{N}^{\prime}\right)=\mathcal{N}$ and hence

$$
c_{R} \leq E_{R}\left[u_{R}, S_{R}\right]=E_{R}^{\prime}\left[u_{R}, S_{R}\right]=c_{R}^{\prime} .
$$

Combining (6.6) and (6.10), we conclude that $c_{R}$ is attained by $\left(u_{R}, S_{R}\right)$. Then we have the following equality:

$$
G_{R}\left[u_{R}, S_{R}\right]=\int_{B_{R}}\left(\left|\nabla u_{R}\right|^{2}+u_{R}^{2}-\beta u_{R}^{2} S_{R}^{2}-u_{R}^{4}\right)=0 .
$$

Hence there exists a Lagrange multiplier $\lambda_{R}$ such that

$$
\nabla E_{R}+\lambda_{R} \nabla G_{R}=0 .
$$

Acting (6.12) on $\left(u_{R}, 0\right)$, we may obtain

$$
\int_{B_{R}}\left(\left|\nabla u_{R}\right|^{2}+u_{R}^{2}-\beta u_{R} S_{R}^{2}-u_{R}^{4}\right)+2 \lambda_{R} \int_{B_{R}}\left(\left|\nabla u_{R}\right|^{2}+u_{R}^{2}-\beta u_{R}^{2} S_{R}^{2}-2 u_{R}^{4}\right)=0,
$$

and hence by (6.11),

$$
-\lambda_{R} \int_{B_{R}} u_{R}^{4}=0, \quad \text { i.e. } \quad \lambda_{R}=0 .
$$

Therefore, we may complete the proof of Lemma 6.1.

Theorem 1.2 is proved by the following lemma

LEMMA 6.2. As $R \rightarrow+\infty,\left(u_{R}, S_{R}\right) \rightarrow\left(u_{\infty}, S_{\infty}\right)$ and $\left(u_{\infty}, S_{\infty}\right)$ is a solution of (1.16).

Proof: Since $S_{R} \leq 1, S_{R}(0)=0$, we first show that $u_{R}$ is uniformly bounded, independent of $R>1$. Actually, it is sufficient to show that $\int_{B_{R}}\left(\left|\nabla u_{R}\right|^{2}+u_{R}^{2}\right) \leq C$, where $C$ is a positive constant independent of $R>1$. Let

$$
G L_{B_{R}}[S]=\int_{B_{R}}\left(\frac{1}{2}|\nabla S|^{2}+\frac{d^{2}}{2 r^{2}} S^{2}+\frac{1}{4}\left(1-S^{2}\right)^{2}\right) .
$$


It is remarkable that $G L_{B_{R}}$ may come from the conventional Ginzburg-Landau functional.

From [21], we may set $\bar{S}_{R}$ as the unique minimizer of $G L_{R}$. Then for any $u \in H_{0}^{1}\left(B_{R}\right)$, there exists $t_{R}$ such that $\left(\sqrt{t_{R}} u, \bar{S}_{R}\right) \in \mathcal{N}$, where $t_{R}$ is simply given by

$$
t_{R}=\frac{\int_{B_{R}}\left(|\nabla u|^{2}+u^{2}-\beta \bar{S}_{R}^{2} u^{2}\right)}{\int_{B_{R}} u^{4}} .
$$

Thus by Lemma 6.1 and $0 \leq \bar{S}_{R}<1$,

$$
\begin{aligned}
c_{R} \leq E_{R}\left[\sqrt{t_{R}} u, \bar{S}_{R}\right] & =G L_{B_{R}}\left(\bar{S}_{R}\right)+\frac{1}{4}\left[\frac{\int_{B_{R}}|\nabla u|^{2}+u^{2}-\beta \bar{S}_{R}^{2} u^{2}}{\left(\int_{B_{R}} u^{4}\right)^{\frac{1}{2}}}\right]^{2} \\
& \leq G L_{B_{R}}\left(\bar{S}_{R}\right)+\left[\frac{\int_{B_{R}}|\nabla u|^{2}+(1-\beta) u^{2}}{\left(\int_{B_{R}} u^{4}\right)^{\frac{1}{2}}}\right]^{2}
\end{aligned}
$$

for all $u \in H_{0}^{1}\left(B_{R}\right)$. Consequently, due to $\beta<0$,

$$
c_{R} \leq G L_{B_{R}}\left(\bar{S}_{R}\right)+C_{0}
$$

where $C_{0}$ is a positive constant independent of $R>1$. Here we have used the fact that $\lim _{R \rightarrow \infty} \inf _{u \in H_{0}^{1}\left(B_{R}\right)} \frac{\int_{B_{R}}|\nabla u|^{2}+(1-\beta) u^{2}}{\left(\int_{B_{R}} u^{4}\right)^{\frac{1}{2}}}<\infty$.

By standard theory of Ginzburg-Landau equation (cf. [1]), we have

$$
G L_{B_{R}}\left[S_{R}\right] \geq G L_{B_{R}}\left[\bar{S}_{R}\right]
$$

Combining (6.14) and (6.15), we see that

$$
\frac{1}{2} \int_{B_{R}}\left(\left|\nabla u_{R}\right|^{2}+u_{R}^{2}-\beta S_{R}^{2} u_{R}^{2}\right)-\frac{1}{4} \int_{B_{R}} u_{R}^{4} \leq C_{3}
$$

and hence by the equation of $u_{R}$,

$$
\int_{B_{R}}\left(\left|\nabla u_{R}\right|^{2}+u_{R}^{2}\right) \leq C
$$

from which standard elliptic regularity theory gives that $u_{R} \leq C$. Thus we may obtain that $\left(u_{R}, S_{R}\right) \rightarrow\left(u_{\infty}, S_{\infty}\right)$ which solves $\Delta u_{\infty}-u_{\infty}+u_{\infty}^{3}+\beta u_{\infty} S_{\infty}^{2}=0$. Note that $u_{R}(0) \geq 1$ and hence $u_{\infty} \not \equiv 0$. By the Maximum Principle, $u_{\infty}>0$. Similarly, $0<S_{\infty}(r)<1$ for $r>0$. Therefore we may complete the proof of Theorem 1.2 . 


\section{Proof of Theorem 1.3}

In this section, we want to construct $S^{2}$-valued map to get half-skyrmions by spike-vortex solutions obtained in Theorem 1.1 and 1.2. For simplicity, we firstly use spike-vortex solutions in Theorem 1.2 to find half-skyrmions. Let $(u, v)$ be the radial spike-vortex solution in Theorem 1.2. We may define a $S^{2}$-valued map by

$$
\vec{n} \equiv \frac{1}{\sqrt{u^{2}+v_{1}^{2}+v_{2}^{2}}}\left(\begin{array}{l}
v_{1} \\
v_{2} \\
u
\end{array}\right)=\left(\begin{array}{l}
\cos (\phi(r)) \cos (d \theta) \\
\cos (\phi(r)) \sin (d \theta) \\
\sin (\phi(r))
\end{array}\right),
$$

where $v=v_{1}+\sqrt{-1} v_{2}$,

$$
\cos (\phi(r))=\frac{f(r)}{\sqrt{u^{2}+f^{2}}}
$$

and

$$
\sin (\phi(r))=\frac{u(r)}{\sqrt{u^{2}+f^{2}}} .
$$

Since both $u$ and $f$ are positive everywhere, the function $\phi$ is well-defined and single-valued. The map $\vec{n}$ can be decomposed into

$$
\vec{n}=\cos (\phi(r))\left(\begin{array}{l}
\cos (d \theta) \\
\sin (d \theta) \\
0
\end{array}\right)+\sin (\phi(r))\left(\begin{array}{l}
0 \\
0 \\
1
\end{array}\right) .
$$

Then it is easy to check that

$$
\begin{aligned}
\int_{\mathbb{R}^{2}} \vec{n} \cdot\left(\partial_{x} \vec{n} \wedge \partial_{y} \vec{n}\right) d x d y & =\int_{\mathbb{R}^{2}}-\frac{d}{r} \phi^{\prime}(r) \cos (\phi(r)) d x d y \\
& =-\left.2 \pi d \sin (\phi(r))\right|_{r=0} ^{+\infty}=2 \pi d,
\end{aligned}
$$

i.e. the topological charge

$$
Q=\frac{1}{4 \pi} \int_{\mathbb{R}^{2}} \vec{n} \cdot\left(\partial_{x} \vec{n} \wedge \partial_{y} \vec{n}\right) d x d y=\frac{d}{2} .
$$

Here we have used (7.3) and the fact that $u(0)>0, u(+\infty)=f(0)=0$ and $f(+\infty)=1$.

For the spike-vortex solution $(u, v)$ in Theorem 1.1. since $\beta>0$ sufficiently small, the associated map $\vec{n}$ has the following form

$$
\vec{n} \equiv \frac{1}{\sqrt{u^{2}+v_{1}^{2}+v_{2}^{2}}}\left(\begin{array}{l}
v_{1} \\
v_{2} \\
u
\end{array}\right)=\left(\begin{array}{l}
\cos (\phi) \cos (d \psi) \\
\cos (\phi) \sin (d \psi) \\
\sin (\phi)
\end{array}\right)
$$

where $\phi=\phi(r, \theta)$ and $\psi=\psi(r, \theta)$ satisfying

$$
\cos (\phi(r, \theta))=\frac{|v|(r, \theta)}{\sqrt{u^{2}+|v|^{2}}}
$$




$$
\sin (\phi(r, \theta))=\frac{u(r, \theta)}{\sqrt{u^{2}+|v|^{2}}},
$$

and $\psi=\theta+h$, where $h$ is a single-valued regular function satisfying $h=O(\beta)$ as $\beta \rightarrow 0+$. Here both $u$ and $|v|$ may not have radial symmetry. Due to $\beta>0$, we may apply the standard maximum principle on the first equation of the system (1.5) i.e. the equation of $u$. Then the solution $u$ is positive everywhere so the function $\phi$ is well-defined and single-valued.

Now we want to calculate the topological charge $Q$ as for (7.4). By (7.5), it is easy to check that

$$
\vec{n} \cdot\left(\partial_{x} \vec{n} \wedge \partial_{y} \vec{n}\right)=-\frac{d}{r} \phi_{r} \cos \phi+\frac{d}{r}\left(\phi_{\theta} h_{r}-\phi_{r} h_{\theta}\right) \cos \phi
$$

Hence by (7.6), (7.7) and using integration by part, we may obtain

$$
Q=\frac{1}{4 \pi} \int_{\mathbb{R}^{2}} \vec{n} \cdot\left(\partial_{x} \vec{n} \wedge \partial_{y} \vec{n}\right)=\frac{d}{2}
$$

Here we have used the fact that $u(0)>0, u(\infty)=0, v(0)=0$ and $|v(\infty)|=1$. Therefore we may complete the proof of Theorem 1.3 .

\section{References}

[1] F. Bethuel, H. Brezis and F. Helen, Ginzburg-Landau vortices. Progress in Nonlinear Differential Equations and their Applications, 13, Birkhauser Boston, Inc., Boston, MA, 1994.

[2] R. A. Battye, N. R. Cooper, and P. M. Sutcliffe, Stable skyrmions in two-component Bose-Einstein condensates, Phys. Rev. Lett. 88 (2002), 080401(1-4).

[3] S. L. Cornish, S. T. Thompson and C. E. Wieman, Formation of bright matter-wave solitons during the collapse of Bose-Einstein condensates, arXiv:cond-mat/0601664.

[4] J. M. Cornwall, Speculations on primordial magnetic helicity, Phys. Rev. D 56, 6146-6154 (1997).

[5] D.Chae and O.Y.Imanuvilov, The existence of non-topological multivortex solutions in the relativistic self-clual Chern-Simons theory, Comm. Math. Phys. 215(2000), 119-142.

[6] B. A. Dubrovin, A. T. Fomenko, S. P. Novikov, Modern Geometry-Methods and Applications, Part II The Geometry and Topology of Manifolds, Springer-Verlag New York, 1985.

[7] F. T. Hioe, Solitary Waves for N Coupled Nonlinear Schrödinger Equations, Phys. Rev. Lett. 82 (1999), 1152(1-4)

[8] F. T. Hioe, T. S. Salter, Special set and solutions of coupled nonlinear schrödinger equations, J. Phys. A: Math. Gen. 35 (2002) 8913-8928. 
[9] T. Kanna and M. Lakshmanan, Exact soliton solutions, shape changing collisions, and partially coherent solitons in coupled nonlinear Schrödinger equations, Phys. Rev. Lett. 86 (2001) 5043(1-4).

[10] E. Lieb and M. Loss, Analysis, American Mathematical Society, 1996.

[11] F. Lin, Static and moving vortices in Ginzburg-Landau theories, Nonlinear partial differential equations in geometry and physics (Knoxville, TN, 1995), 71-111, Progr. Nonlinear Differential Equations Appl., 29, Birkhauser, Basel, 1997.

[12] F. Lin and T. C. Lin, Vortices in two-dimensional Bose-Einstein condensates. Geometry and nonlinear partial differential equations (Hangzhou, 2001), 87-114, AMS/IP Stud. Adv. Math., 29, Amer. Math. Soc., Providence, RI (2002).

[13] F. Lin and Y. Yang, Existence of two-dimensional skyrmions via the concentrationcompactness method, Comm. Pure Appl. Math. 57 (2004), no. 10, 1332-1351.

[14] T.C. Lin, The stability of the radial solution to the Ginzburg-Landau equation, Comm. PDE 22 (1997), no. 3-4, 619-632.

[15] T. C. Lin and J. Wei, Spikes in two coupled nonlinear Schr ödinger equations, Ann. I. H. Poincaré Analyse. Non. 22, no.4(2005) 403-439.

[16] T.C.Lin and J.C.Wei, Ground state of $N$ coupled nonlinear Schrodinger equations in $\mathbb{R}^{n}$, $n \leq$ 3. Comm. Math. Phys. 255 (2005), no. 3, 629-653; Erratum, Comm. Math. Phys., to appear.

[17] V. Makhankov et al., The Skyrme Model (Springer-Verlag, New York, 1993).

[18] T. Morinari, Half-skyrmion picture of a single-hole-doped $\mathrm{CuO}_{2}$ plane, Phys. Rev. B 72, 104502(1-10) (2005).

[19] W.-M. Ni, Qualitative properties of solutions to elliptic problems. Stationary partial differential equations. Vol. I, 157-233, Handb. Differ. Equ., North-Holland, Amsterdam, 2004.

[20] W.-M. Ni and I. Takagi, Locating the peaks of least energy solutions to a semilinear Neumann problem, Duke Math. J. 70(1993), 247-281.

[21] F. Pacard, T. Riviere, Linear and nonlinear aspects of vortices, the Ginzburg-Landau model, progress in nonlinear differential equations and their applications, 39(2000), Birkhäuser, Boston.

[22] L. Pitaevskii and S. Stringari, Bose-Einstein condensation, Oxford 2003

[23] W. C. Stwalley, Stability of Spin-Aligned Hydrogen at Low Temperatures and High Magnetic Fields: New Field-Dependent Scattering Resonances and Predissociations, Phys. Rev. Lett. 37, 1628 (1976). 
[24] E.Tiesinga, A. Moerdijk, B. J. Verhaar and H. T. C. Stoof, Conditions for Bose-Einstein condensation in magnetically trapped atomic cesium, Phys. Rev. A 46, 1167-1170 (1992).

[25] C.M. Savage1, and J. Ruostekoski, Energetically Stable Particlelike Skyrmions in a Trapped Bose-Einstein Condensate, Phys. Rev. Lett. $91010403(1-4)$ (2003).

[26] S. Wuster, T. E. Argue, and C. M. Savage, Numerical study of the stability of skyrmions in Bose-Einstein condensates, Phys. Rev. A 72, 043616(1-8) (2005). 